\title{
Monoclonal Antibody m170
}

National Cancer Institute

\section{Source}

National Cancer Institute. Monoclonal Antibody m170. NCI Thesaurus. Code C1959.

A panadenocarcinoma murine monoclonal antibody (MoAb) with potential antineoplastic activity. MoAb m170 recognizes MUC-1 antigen present on the surface of many adenocarcinomas. It may be conjug ated with a radioactive element and used in radioimmunotherapy (RIT), a procedure that uses a tumor-specific monoclonal antibody to target radiation to cancer cells. 